\title{
Optimal design of the air-balancing device for the rear arm of anti
}

\section{terrorist and riot robot}

\author{
Zhang Xinglei ${ }^{1,3, a},{ }^{*}$ Fan Binghui ${ }^{2, b}$, Liu Hanjin ${ }^{1, c}$ \\ ${ }^{1}$ College of Mechanical and Electrical Engineering, Shandong University of Science and \\ Technology, Qingdao 266590, China; \\ ${ }^{2}$ College of Electrical Engineering and Automation, Shandong University of Science and \\ Technology, Qingdao 266590, China \\ ${ }^{3}$ Department of Mechanical and Electrical Engineering , Zaozhuang University , Zaozhuang \\ 277160, China \\ azhangxingfulove@163.com, bfanbh58@163.com, 411366763@qq.com
}

\begin{abstract}
Keywords: manipulator, residual torque, MATLAB,optimization,air-balance device
\end{abstract}
Abstract. Anti terrorist and riot robot is composed of laser guidance, grasping system and walking device. It can replace some related personnel to do some dangerous operations. The grasping system studied in this paper is mainly composed of four parts: base, rear arm, forearm and mechanical gripper. Joint rotation and mechanical gripper are driven by steering gear. In order to overcome the heavy torque produced by the mechanical arm and the external load in the working process, the steering engine is required to provide greater driving force. In order to reduce the driving torque of joint steering engine and improve limit load of the manipulator. This paper put forward to install of air-balancing device on the robot rear arm, and determine the optimal variables, constraints and objective function. By using MATLAB software for optimization analysis, the best installation position, optimal force of the air-balancing device and the simulation figure about output motion are obtained, and the correctness of the optimization results is verified.

\section{Introduction}

In order to deal with the threat of an explosion in a terrorist organization, it is necessary to take effective and safe measures, but sometimes it needs to investigate suspicious items. If the dispatch of human resources is often caught in a trap and causes the sacrifice of professionals, the foreign police organization always uses robots to check suspicious items. In foreign countries, the robot handling of such events is quite common. At home, the related departments with anti terrorist and riot robots are less ${ }^{[1]}$. Anti terrorist and riot robots can perform some dangerous jobs instead of related personnel, and can be used to search, detect and deal with all kinds of explosive dangerous $\operatorname{goods}^{[2]}$.

The prototype of the anti terrorist and riot robot manipulator designed in this paper is shown in Figure 1, and it includes the base torsional joint, the rear arm pitch joint, the forearm pitch joint, the wrist torsion joint, the wrist pitch joint and the claw opening joint. In order to reduce the driving torque required by the joint motor of manipulator, this paper proposes to balance the mechanical arm and the load torque with the air-balance device in order to reduce the driving power requirement of the steering gear. 


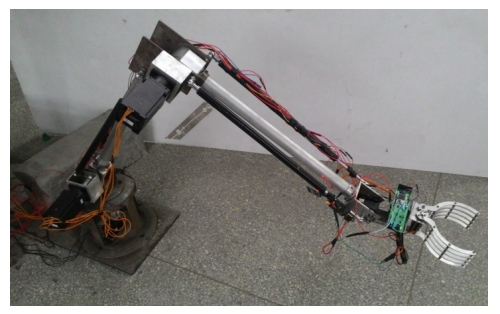

Fig. 1 anti terrorist and riot robot manipulator

The schematic diagram of the overall structure of a manipulator is shown in Figure 2. The installation structure at both ends of the air-balancing device is a form of ball hinges. By using the air-balancing device, the tension of the device is basically unchanged during the expansion process, and the position of the air-balancing device and the size of the tension are reasonably selected. When the arm is pitching, the air- balancing device will expand and change. Then the torque produced by the air- balance device can balance the torque of the manipulator and the load to a large extent, so that the driving torque of the steering arm needed by the rotating arm will be greatly reduced.

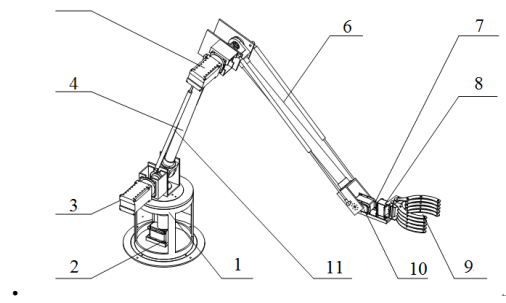

1.manipulator base 2. pedestal steering gear 3. rear arm pitch motor 4.rear arm5.fore-arm pitch motor 6.fore-arm 7.wrist 8.wrist rotation motor 9.mechanical gripper10.wrist pitch motor 11.air-balancing device

Fig .2 the structure of the manipulator

\section{Optimal design}

\section{Build mathematical models}

Robotic rear arm air-balance structure mathematical model is shown in figure 3. It is known that three parameters are required to complete the installation of the rear arm air-balancing device, numbered $x_{1}, x_{2}, x_{3}$, The $x_{1}$ is the tension of the air-balancing device. The $x_{2}$ is the vertical distance of the lower hinge connection to the rotating center of the rudder of the air-balancing device. That is $O M$ in the diagram. The $x_{3}$ is the connection distance between the hinge point of the air-balancing device and the rotation center of the large arm rudder. And that's $O N$, The center gravity of the rear arm 、 forearm-hand and the load is $G_{1}, G_{2}, G_{3}$ respectively. The range of $\alpha$ for the pitch motion angle of the rear arm is $\left[30^{\circ}, 150^{\circ}\right]$. The range of the angle between the forearm and the rear arm is $\left[30^{\circ}, 150^{\circ}\right]$ 


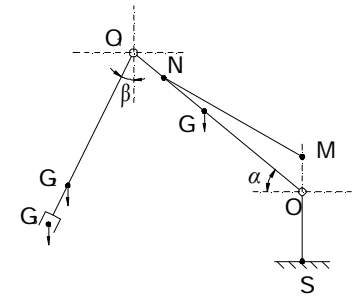

Fig .3 the installation principle of the large arm balancing device

In the range of the arm movement, we set up the balance moment of the air- balancing device of the rear $\operatorname{arm} M_{P}$, the rear arm pitching moment $M_{d}$, the forearm and hand heavy torque $M_{x}$, the load of gravity moment $M_{f}$ :

$$
\begin{gathered}
L_{d}=O G_{1} \cdot \cos \alpha \\
L_{\mathrm{x}}=O O_{1} \cdot \cos \alpha+O_{1} G_{2} \cdot \sin \beta \\
M N=\sqrt{x_{2}{ }^{2}+x_{3}{ }^{2}-2 \cdot x_{2} \cdot x_{3} \cdot \cos \left(\frac{\pi}{2}-\alpha\right)} \\
L_{p}=\frac{x_{2} \cdot x_{3} \cdot \sin \left(\frac{\pi}{2}-\alpha\right)}{M N} \\
L_{f}=O O_{1} \cdot \cos \alpha+O_{1} G_{3} \cdot \sin \beta \\
M_{p}=2 \cdot x_{1} \cdot L_{p} \\
M_{d}=m_{1} \cdot g \cdot L_{d} \\
M_{x}=m_{2} \cdot g \cdot L_{x} \\
M_{f}=m_{3} \cdot g \cdot L_{f}
\end{gathered}
$$

In the diagram, $L_{d}$ rear arm gravity arm,$L_{x}$ :forearm and hand gravity arm , $L_{p}$, rear arm balance device force arm , $L_{f}:$ load gravity arm , $\alpha$ : the pitching angle of the rear arm , $\beta$ : the pitching angle of the forearm , $g$ :gravitational acceleration , $m_{1}$ :mass of the rear arm , $m_{2}$ :mass of forearm and hand, $m_{3}$ : mass of load.

\section{Determine the constraint function}

According to the structural space of Manipulator, the working performance of air-tie rod and the interference of motion process, the constraint function of each design variable is given:

$$
0 \leq x_{1} \leq 1000 \mathrm{~N}, \quad 10 \mathrm{~mm} \leq x_{2} \leq 100 \mathrm{~mm}, \quad 10 \mathrm{~mm} \leq x_{3} \leq 500 \mathrm{~mm}
$$




\section{Determine the objective function}

The maximum absolute value of residual torque is the objective function when the robotic arm grabs the load in pitching process, and then the objective function is optimized to get the minimum.

$$
\mathrm{f}(\mathrm{x})=\max \left(\operatorname{abs}\left(\Delta M_{1}\right)\right)
$$

In the formula $\Delta M_{1}=M_{\mathrm{di}}+M_{\mathrm{xi}}+M_{\mathrm{fi}}-M_{\mathrm{pi}}(i=1,2, \mathrm{~L} 11)$ is residual torque at different angles When the rear arm is pitching. $f(x)$ is the objective function, which expresses the maximum value of the $\Delta M_{1}$ absolute value at each discrete position of a manipulator motion in a load state; and the result of the optimal design is to minimize the $f(x)$.

\section{Optimization results}

In order To meet the requirements of compact space, small driving torque and low energy consumption of the manipulator structure, , the optimal design procedure is programmed in the MATLAB environment to achieve the best selection of all design variables ${ }^{[3-4]}$. In this paper, $m_{1}=2.4 \mathrm{~kg} ; \mathrm{m}_{2}=2.0 \mathrm{~kg} ; m_{3}=5 \mathrm{~kg} ; O O_{1}=O_{1} G_{3}=550 \mathrm{~mm} ; O_{1} G_{2}=410 \mathrm{~mm}$, With the help of nonlinear constrained solving function fmincon in MATLAB, We get the best installation parameters of dynamic balance device of the robotic arm and the gas balance force. The result of optimization is:

$$
x_{1}=228 \mathrm{~N}, x_{2}=80 \mathrm{~mm}, x_{3}=490 \mathrm{~mm}, \mathrm{f}=30 \mathrm{Nm}
$$

According to the optimization results, editing motion simulation program ${ }^{[5]}$ is used by the MATALB to get all the attitude of the pitch motion of the manipulator, as shown in Figure 4.Robotic arm motor driving torque is shown in figure 5, the results show that at rated load condition, the rear arm motor torque maximum is $30 \mathrm{Nm}$ in the state of installed of air-balance device ,but the rear arm motor torque maximum is rising to $75 \mathrm{Nm}$ after uninstalled of air-balance device. The air-balance device can balance the manipulator and heavy load torque of more than $60 \%$ in its working range, and it greatly reduces the steering engine required driving torque.

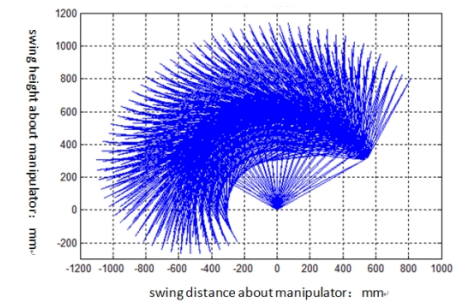

Fig.4 demonstration of the manipulator

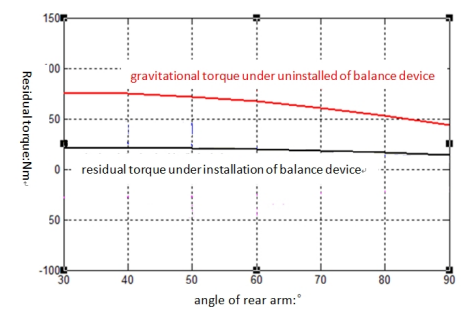

Fig.5 residual torque curve under rated load state 


\section{Conclusions}

In the movement process of a anti terrorist and riot robot, in order to reduce the driving torque of joint steering engine and improve limit load of the manipulator. This paper put forward to install of air-balancing device on the robot rear arm, and the best installation position, optimal force of the air-balancing device are obtained by using MATLAB software for optimization analysis. It is clear that the balance effect of the installation balance device is obvious by comparing the driving torque simulation diagram of the steering gear in two states. So, the balancing device installed in robotic arm is of great significance.

\section{Acknowledgements}

This research was supported by two foundation projects:1. Key research and development project of Shandong province (2016GSF201197); 2. Project of science and technology project of Shandong higher education institution (J16LB11).

\section{References}

[1] Fu Wei, Liu Meijun. Research and development of anti terrorism anti riot robot [M]. Beijing: Electronic Industry Press ,2010,1-3.

[2] D Whitley.An Executable Model of a Simple Genetic Algorithm[J].Foundations of Genetic Algorithms, 1993,2:45-62.

[3] MA Ahmadi,S Zendehboudi,A Lohi,A Elkamel,I Chatzis.Reservoir permeability prediction by neural networks combined with hybrid genetic algorithm and particle swarm optimization[J].19. Geophysical Prospecting, 2013,61(3):582-598.

[4] Ma Changfeng, optimization method and Matlab program design [M]. Beijing: Science Press, 2010.

[5] Wang Zhixing, Fan Wenxin, Zhang Baocheng, et al. Kinematic analysis and Simulation of industrial robots based on Matlab [J]. mechanical and electrical engineering, 2012, 29 (1): 33-37. 